\title{
QseBC is involved in the biofilm formation and antibiotic resistance in Escherichia coli isolated from bovine mastitis
}

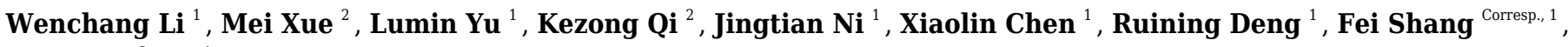 \\ Ting Xue ${ }^{\text {Corresp. } 1}$ \\ ${ }^{1}$ School of Life Sciences, Anhui Agricultural University, Hefei, Anhui, China \\ 2 Anhui Province Key Laboratory of Veterinary Pathobiology and Disease Control, Anhui Agricultural University, Hefei, Anhui, China \\ Corresponding Authors: Fei Shang, Ting Xue \\ Email address: shf@ahau.edu.cn, xuet@ahau.edu.cn
}

\section{Background}

Mastitis is one of the most common infectious diseases in dairy cattle and causes significant financial losses in the dairy industry worldwide. Antibiotic therapy has been used as the most effective strategy for clinical mastitis treatment. However, due to the extensive use of antibacterial agents, antimicrobial resistance (AMR) is considered to be one of the reasons for low cure rates in bovine mastitis. In addition, biofilms could protect bacteria by restricting antibiotic access and shielding the bacterial pathogen from mammary gland immune defences. The functional mechanisms of quorum sensing $E$. coli regulators $B$ an d C (QseBC) have been well studied in E. coli model strains; however, whether QseBC regulates antibiotic susceptibility and biofilm formation in clinical $E$. coli strain has not been reported.

\section{Methods}

In this study, we performed construction of the $q s e B C$ gene mutant, complementation of the $q s e B C$ mutant, antimicrobial susceptibility testing, antibacterial activity assays, biofilm formation assays, realtime reverse transcription PCR (RT-PCR) experiments and electrophoretic mobility shift assays (EMSAs) to investigate the role of $q s e B C$ in regulating biofilm formation and antibiotic susceptibility in the clinical $E$. coli strain ECDCM2.

\section{Results}

We reported that inactivation of QseBC led to a decrease in biofilm formation capacity and an increase in antibiotic susceptibility of an $E$. coli strain isolated from a dairy cow that suffered from mastitis . In addition, this study indicated that QseBC increased biofilm formation by upregulating the transcription of the biofilm-associated genes bcsA, csgA, flic, motA, wcaF and fimA and decreased antibiotic susceptibility by upregulating the transcription of the efflux-pump-associated genes marA, acrA, acrB, $a c r D$, emrD and $m d t H$. We also performed EMSA assays, and the results showed that QseB can directly bind to the marA promoter.

\section{Conclusions}

The QseBC two-component system affects antibiotic sensitivity by regulating the transcription of effluxpump-associated genes. Further, biofilm-formation-associated genes were also regulated by QseBC TCS in E. coli $\mathrm{ECDCM} 2$. Hence, this study might provide new clues to the prevention and treatment of infections caused by the clinical $E$. coli strains. 
1 SHORT TITLE: Regulatory role of QseBC in E. coli

2

3 QseBC is involved in biofilm formation and antibiotic resistance in Escherichia coli isolated

4 from bovine mastitis

5

6 Wenchang $\mathbf{L i}^{*}$, Mei Xue ${ }^{\dagger}$, Lumin Yu*, Kezong Qi†, Jingtian Ni*, Xiaolin Chen*, Ruining

7 Deng*, Fei Shang*,1, Ting Xue ${ }^{*}, 1$

8

9 *School of Life Sciences, Anhui Agricultural University, Hefei, Anhui 230036, China

$10 \uparrow^{\dagger}$ Anhui Province Key Laboratory of Veterinary Pathobiology and Disease Control, Anhui

11 Agricultural University, Hefei, Anhui 230036, China.

12

$13{ }^{1}$ Corresponding author.

14 Tel: (86) 551 65787380; Fax: (86) 551 65787380;E-mail: shf@ahau.edu.cn

15 Tel: (86) 551 65787380; Fax: (86) 551 65787380; E-mail: xuet@ahau.edu.cn 
ABSTRACT

\section{Background}

Mastitis is one of the most common infectious diseases in dairy cattle and causes significant financial losses in the dairy industry worldwide. Antibiotic therapy has been used as the most effective strategy for clinical mastitis treatment. However, due to the extensive use of antibacterial agents, antimicrobial resistance (AMR) is considered to be one of the reasons for low cure rates in bovine mastitis. In addition, biofilms could protect bacteria by restricting antibiotic access and shielding the bacterial pathogen from mammary gland immune defences. The functional mechanisms of quorum sensing E. coli regulators B and C (QseBC) have been well studied in $E$. coli model strains; however, whether QseBC regulates antibiotic susceptibility and biofilm formation in clinical E. coli strain has not been reported.

\section{Methods}

In this study, we performed construction of the qseBC mutant, complementation of the qseBC mutant, antimicrobial susceptibility testing, antibacterial activity assays, biofilm formation assays, real-time reverse transcription PCR (RT-PCR) experiments and electrophoretic mobility shift assays (EMSAs) to investigate the role of $q s e B C$ in regulating biofilm formation and antibiotic susceptibility in the clinical E. coli strain ECDCM2.

\section{Results}

We reported that inactivation of QseBC led to a decrease in biofilm formation capacity and an increase in antibiotic susceptibility of an E. coli strain isolated from a dairy cow that suffered from mastitis. In addition, this study indicated that QseBC increased biofilm formation by upregulating 
38 the transcription of the biofilm-associated genes $b \operatorname{cs} A, \operatorname{csg} A, f l i C, m o t A, w c a F$ and $f i m A$ and

39 decreased antibiotic susceptibility by upregulating the transcription of the efflux-pump-associated

40 genes $\operatorname{mar} A, a c r A, a c r B, a c r D$, emrD and $m d t H$. We also performed EMSA assays, and the results

41 showed that QseB can directly bind to the marA promoter.

\section{Conclusions}

43 The QseBC two-component system affects antibiotic sensitivity by regulating the transcription of

44 efflux-pump-associated genes. Further, biofilm-formation-associated genes were also regulated by

45 QseBC TCS in E. coli ECDCM2. Hence, this study might provide new clues to the prevention and treatment of infections caused by the clinical E. coli strains.

Keywords: Escherichia coli; bovine mastitis; QseBC two-component system; efflux pump; biofilm 
52 Among many diseases present in dairy cows, mastitis has always occupied the top of the pyramid

53 as the most common and economically severe disease affecting dairy cattle throughout the world

54 (Saini et al. 2012; Yang et al. 2016). A variety of microorganisms have been isolated from bovine

55 mastitis cases. In fact, 137 different microorganisms were identified by Watts in 1988 from

56 mastitis cases, and among them, five species of etiological agents were the most common causes

57 of mastitis, including Escherichia coli, Staphylococcus aureus, Streptococcus agalactiae,

58 Streptococcus uberis and Streptococcus dysgalactiae (Nair et al. 2005). The incidence of

59 Escherichia coli, the representative environmental pathogen, is little affected by mastitis control

60 programs such as the large-scale utilization of the 5-point plan and improved milking hygiene

61 (Elhadidy \& Elsayyad 2013; Neave et al. 1969).

62 Antibacterial drugs are the mainstay of treatment for mastitis prevention and control. However,

63 their therapeutic effectiveness is limited by the increasing incidence of antimicrobial resistant

64 (AMR) infections (Dias et al. 2013). The activation of an efflux pump has been known to play a

65 predominant role in resistance to certain drugs. So far, members of the resistance-nodulation-cell

66 division (RND) family appear to be the high-efficiency efflux systems in gram-negative bacteria.

67 AcrAB is a multidrug efflux pump of the E. coli of RND family, whose transcription is activated

68 by MarA (Perez et al. 2012; Randall \& Woodward 2002). Previous studies indicated that MarA

69 activates the expression of $a c r A B$ in response to various chemicals in the environment (Baranova

70 \& Nikaido 2002). The major multidrug efflux pump, AcrAB, which extrudes an extremely broad

71 range of antimicrobial compounds, is essential for bacterial survival and colonization (Nikaido \&

Peer] reviewing PDF | (2019:10:42311:2:0:NEW 22 Feb 2020) 
72 Pages 2012). AcrD, an RND-type efflux pump, is a close homolog of AcrB and characterized as a

73 transporter of aminoglycosides (Elkins \& Nikaido 2002; Rosenberg et al. 2000). Furthermore,

74 EmrD and MdtH (Nishino \& Yamaguchi 2001) are members of the major facilitator superfamily

75 (MFS). EmrD is a multidrug efflux protein involved in the efflux of amphipathic compounds and

76 adaptation to low-energy shock in Escherichia coli (Baker et al. 2012; Naroditskaya et al. 1993).

77 The efficacy of antibiotics in the treatment of bacterial infectious diseases is heavily affected by

78 the emergence of multidrug resistance. Additionally, the use of antibiotic abusively and non-

79 normatively may be a potentially important driver of the formation of AMR.

80 Two-component systems are essential for bacteria to quickly respond and adapt to various

81 environmental stimuli, particularly through changes in target gene transcription (Beier \& Gross

82 2006). In the QseBC two component system, the environmental signal bacterial autoinducer 3 (AI-

83 3) and the mammalian stress hormones epinephrine and norepinephrine (Clarke et al. 2006;

84 Hughes et al. 2009; Sharma \& Casey 2014) are sensed by QseC (an inner membrane-bound sensor

85 histidine kinase $(\mathrm{HK})$ ), which initiates a signal transduction cascade that, in many cases, results in

86 the phosphorylation of aspartate residue of QseB (the response regulator (RR)) (Hoch \&

87 Varughese 2001; Weigel \& Demuth 2016). QseB regulates the transcription of downstream target

88 genes. QseBC TCS was first discovered in enterohaemorrhagic Escherichia coli (EHEC) and

89 generally functions as a global regulator of virulence (Weigel \& Demuth 2016). Besides, other

90 studies have demonstrated that QseBC TCS contributes to the regulation of colonization, motility

91 and expression of virulence genes in Enterohaemorrhagic Escherichia coli O157:H7 (Clarke \&

92 Sperandio 2005b; Sharma \& Casey 2014), motility and biofilm formation in Escherichia coli 
93 MG1655 (Clarke \& Sperandio 2005b; Gou et al. 2019), bacterial adherence in Actinobacillus

94 pleuropneumoniae (Liu et al. 2015) and biofilm formation in Aggregatibacter

95 actinomycetemcomitans (Juarez-Rodriguez et al. 2013). However, whether QseBC regulate the

96 antibiotic susceptibility and biofilm formation in E. coli strain isolated from bovine mastitis has

97 not been reported. In this study, we investigated the effects of QseBC on antibiotic susceptibility

98 and biofilm formation and further explored the mechanisms of how QseBC regulates antibiotic

99 susceptibility and biofilm formation in the clinical E. coli strain ECDCM2.

100

101

\section{MATERIALS AND METHODS}

\section{Bacterial strains, plasmids and growth conditions}

103 The bacterial strains and plasmids used in this study are listed in Table 1. Escherichia coli isolated

from a dairy cow with mastitis (ECDCM2) was routinely grown in Luria-Bertani (LB) broth with

shaking at $150 \mathrm{rpm}$ or on solid medium without shaking. Temperature-sensitive plasmids pKD46

or pCP20 were maintained at $30^{\circ} \mathrm{C}$. The appropriate antibiotics for plasmid selection and

maintenance were used at the following final concentrations: chloramphenicol at $16 \mu \mathrm{g} / \mathrm{ml}$ and

ampicillin at $100 \mu \mathrm{g} / \mathrm{ml}$.

\section{Construction of the qseBC mutant}

110 The qseBC-deficient mutant strain was constructed by using homologous recombination methods

111 based on the $\lambda$ red recombinase system (Datsenko \& Wanner 2000). Briefly, WT strain competent

112 cells were transformed with the $\lambda$ red recombinase system plasmid pKD46, and incubated in LB

113 broth with $100 \mu \mathrm{g} / \mathrm{ml}$ of ampicillin at $30^{\circ} \mathrm{C}$ with shaking at $150 \mathrm{rpm}$ to an $\mathrm{OD}_{600}$ of approximately 
114 0.3. A final concentration of $20 \mathrm{mM}$ of L-arabinose (Sangon, Shanghai, China) was then added to

115 the cultures, which were cultured continuously at $30^{\circ} \mathrm{C}$ with shaking at $150 \mathrm{rpm}$ to an $\mathrm{OD}_{600}$ of

116 approximately 0.6 . Subsequently, electrocompetent cells were made with ice-cold, sterilized 10\%

117 glycerol. The chloramphenicol-resistance cassette (cat), flanked by 40-base-pair homology arms

118 located upstream and downstream of the $q s e B C$ genes, was amplified by PCR using pKD3 as a

119 template with the primers knockout-qseBC-f and knockout-qseBC-r. Then, PCR products were

120 gel-purified and dissolved with sterilized distilled deionized water $\left(\mathrm{ddH}_{2} \mathrm{O}\right)$. The purified PCR

121 products were transformed into electrocompetent cells by using Gene Pulser XcellTM 154 (Bio-

122 Rad, Hercules, California, USA) at $2.5 \mathrm{kV}, 200 \Omega$ and $25 \mu \mathrm{F}$. After electroporation, shocked cells

123 were recovered in $500 \mu \mathrm{L}$ LB broth without antibiotics and incubated at $37^{\circ} \mathrm{C}$ for $1.5 \mathrm{~h}$ with

124 shaking at $150 \mathrm{rpm}$, then spread on LB agar containing chloramphenicol to select for resistant

125 mutants. After incubation overnight, chloramphenicol-resistant colonies were picked and

126 inoculated into LB broth containing $16 \mu \mathrm{g} / \mathrm{ml}$ chloramphenicol. The presence of the mutant was

127 confirmed by PCR using primers check-qseBC-f and check-qseBC-r. The mutant colonies were

128 inoculated into fresh LB broth with $16 \mu \mathrm{g} / \mathrm{ml}$ chloramphenicol and incubated with aeration at $42^{\circ} \mathrm{C}$

129 for $16 \mathrm{~h}$ to remove the pKD46 plasmid. The plasmid pCP20 was then transformed into the mutant

130 strain to cure the cat. Following incubation at $30^{\circ} \mathrm{C}$ overnight, the resulting ampicillin resistant

131 colonies were inoculated into LB broth with $100 \mu \mathrm{g} / \mathrm{ml}$ ampicillin and removed plasmid pCP20 at

$13242^{\circ} \mathrm{C}$ for $48 \mathrm{~h}$. The mutant strain was named XL4. The primers used in this study are listed in Table 1332.

134 Complementation of the qseBC mutant 
135 The full length of the qseBC ORF was amplified by PCR using the chromosomal DNA of the wild-

136 type strain (WT) using primers qseBC-EcoRI-f and qseBC-KpnI-r. The PCR products were gel-

137 purified and ligated into the EcoRI and KpnI sites of the low-copy-number plasmid pSTV28

138 (TaKaRa, Dalian, Liaoning, China). The recombinant plasmid pSTV-qseBC was transformed into

139 E. coli DH5 $\alpha$ chemically competent cells, which were then spread on LB agar with $16 \mu \mathrm{g} / \mathrm{ml}$

140 chloramphenicol. Primers M13-f and M13-r were used to confirm the positive colonies, and then

141 the recombinant plasmid pSTV-qseBC was extracted and further confirmed by DNA sequencing

142 (Fig. S1). Finally, the mutant strain XL4 containing the recombinant plasmid pSTV-qseBC was

143 named XL4/pCqseBC. As a control, WT and XL4 were also transformed with the empty vector

144 pSTV28 and renamed as WT/pSTV28 and XL4/pSTV28, respectively.

\section{Bacterial Growth Curves}

146 Growth curves of WT/pSTV28, XL4/pSTV28, and XL4/pCqseBC were monitored, as described

147 previously, with some modifications (Xue et al., 2016). Briefly, overnight cultures of

$148 \mathrm{WT} / \mathrm{pSTV} 28, \mathrm{XL4} / \mathrm{pSTV} 28$ and XL4/pCqseBC were each diluted to an $\mathrm{OD}_{600}$ of approximately

1490.03 in $50 \mathrm{ml}$ of fresh LB broth (Oxoid, Basingstoke, UK) and grown at $37^{\circ} \mathrm{C}$ with shaking for 26

$150 \mathrm{~h}$ with $16 \mu \mathrm{g} / \mathrm{ml}$ chloramphenicol. The cell density was detected every $2 \mathrm{~h}$ using a UV/Vis

151 spectrophotometer (DU730, Beckman Coulter, Miami, FL). Experiments were repeated three

152 times with two replicates.

\section{Antimicrobial susceptibility testing}

154 Strain WT was a clinical E. coli strain isolated from a dairy cow that suffered from mastitis. Broth

155 dilution was used to examine the changes in antimicrobial susceptibility of the WT/pSTV28, 
$156 \mathrm{XL4/pSTV} 28$ and XL4/pCqseBC according to the Clinical Laboratory Standard Institute (CLSI)

157 in the presence of nine kinds of antibiotics. Strains WT/pSTV28, XL4/pSTV28 and XL4/pCqseBC

158 were grown for $16 \mathrm{~h}$ in Mueller-Hinton (MH) broth (Oxoid, Basingstoke, UK) with $16 \mu \mathrm{g} / \mathrm{ml}$

159 chloramphenicol and diluted 1:100 in fresh $\mathrm{MH}$ with $16 \mu \mathrm{g} / \mathrm{ml}$ chloramphenicol. The dilutions

160 were dispensed into a series of Eppendorf (EP) tubes containing serial dilutions of antibiotics with

161 broth dilution. The diluted cultures were incubated at $37^{\circ} \mathrm{C}$ for $24 \mathrm{~h}$ and the MIC determined.

162 Experiments were repeated three times for each group.

\section{Antibacterial activity assays}

164 Antimicrobial activity assays were performed according to a previous study (Chen et al. 2015).

165 Overnight cultures of the isogenic derivative strains (WT/pSTV28, XL4/pSTV28, XL4/pCqseBC)

166 were inoculated into fresh LB broth with $16 \mu \mathrm{g} / \mathrm{ml}$ chloramphenicol and then diluted to a final

167 optical density of 0.03 at $600 \mathrm{~nm}$ in $\mathrm{LB}$ broth and incubated at $37^{\circ} \mathrm{C}$ with shaking at $150 \mathrm{rpm}$ for 3

168 h. Subsequently, gentamicin and ciprofloxacin were added to the test group at final concentrations

169 of $8 \mu \mathrm{g} / \mathrm{ml}$ and $10.7 \mu \mathrm{g} / \mathrm{ml}$, respectively, and the cultures were incubated for $2 \mathrm{~h}$ at $37^{\circ} \mathrm{C}$ with

170 continuous shaking at $150 \mathrm{rpm}$. Next, $100 \mu \mathrm{L}$ aliquots were dropped onto LB agar plates and

171 distributed evenly with a spreader. After culturing for $16 \mathrm{~h}$ at $37^{\circ} \mathrm{C}$, viable colonies were counted

172 and compared among the isogenic derivative strains via their colony-forming units (CFU) on LB

173 agar plates. The survival rate is the ratio of the surviving colony counts of the derivative strains to

174 that of the WT/pSTV28 exposed to antibiotics. Experiments were repeated three times for each

175 group.

176 Biofilm formation assays 
177 Biofilm formation was quantified according to the procedure described elsewhere and modified as

178 described herein (Khajanchi et al. 2012). Briefly, the isogenic derivative strains (WT/pSTV28,

$179 \mathrm{XL4} / \mathrm{pSTV} 28, \mathrm{XL} 4 / \mathrm{pCqseBC})$ were grown overnight in LB broth with $16 \mu \mathrm{g} / \mathrm{ml}$ chloramphenicol

180 and diluted in fresh LB with chloramphenicol. Skim milk was added to the diluted cultures at a

181 final concentration of $0.5 \%$. Cultures were transferred to sterile glass tubes and incubated at $37^{\circ} \mathrm{C}$

182 for $72 \mathrm{~h}$ without shaking. After incubation, the growth medium was removed, and excess bacteria

183 were washed off gently with sterile water and air-dried. Adherent bacteria were stained with $0.1 \%$

184 crystal violet (CV, Sangon, Shanghai, China) for $20 \mathrm{~min}$. Next, the CV was removed; the residual

185 stain was rinsed gently with distilled water and air-dried overnight. Finally, the purple area in the

186 glass tubes was dissolved with 33\% glacial acetic acid (Sangon, Shanghai, China), and the biomass

187 of the biofilm was determined by using a Micro ELISA auto reader (Thermo Scientific, Pittsburgh,

188 PA) at a wavelength of $492 \mathrm{~nm}$ in single-wavelength mode. Absorbance data from three replicate

189 wells were averaged to obtain each data point.

190 Total RNA isolation, cDNA generation and real-time PCR processing

191 Strains WT/pSTV28, XL4/pSTV28 and XL4/pCqseBC were diluted in LB media with $16 \mu \mathrm{g} / \mathrm{ml}$

192 chloramphenicol to a final $\mathrm{OD}_{600}$ of 0.03 and grown to late exponential phase $\left(\mathrm{OD}_{600}=2.5\right)$. Cells

193 were collected and resuspended in sterile EDTA water ( $\mathrm{pH} 8.0)$, total RNA was extracted from

194 cells using TRIzol UP (Transgene, China), and residual DNA was removed using DNase (TaKaRa,

195 Dalian, China). The transcript levels of genes associated with biofilm formation were tested by

196 Quantitative reverse-transcription PCR (RT-qPCR), the and cDNA Synthesis SuperMix kit and

197 TransStart $^{\circledR}$ Tip Green qPCR Supermix kit (Transgene, China) were used according to the 
198

199

200

201

202

203

204

205

206

207

208

209

210

211

212

213

214

215

216

217

218

manufacturer's instructions. Differences in gene expression were calculated by using the $\Delta \Delta^{\mathrm{Ct}}$

(where $\mathrm{Ct}=$ cycle threshold) method, using $16 \mathrm{~S}$ gene as the housekeeping gene, normalized by subtracting the $\mathrm{Ct}$ value of $16 \mathrm{~S}$ cDNA from the target cDNA. All of the real-time RT-PCR assays were repeated at least three times.

\section{Purification of the QseB protein}

The expression vector pET28a (+) (Novagen), which encompassed the sequence of His $_{6}$-tagged QseB was transformed into E. coli BL21 (DE3). The transformants were grown in $100 \mathrm{ml} \mathrm{LB}$ at $37^{\circ} \mathrm{C}$ to an $\mathrm{OD}_{600}$ of approximately 0.3 , transferred to $16^{\circ} \mathrm{C}$, and induced overnight with a final concentration of $0.25 \mu \mathrm{g} / \mathrm{ml}$ IPTG. Cells were collected by centrifugation and washed three times with cell washing buffer (20 mM Na $2 \mathrm{HPO}_{4}, 20 \mathrm{mM} \mathrm{NaH}_{2} \mathrm{PO}_{4}, \mathrm{pH} 7.8$ and $\left.500 \mathrm{mM} \mathrm{NaCl}\right)$, and then the cells were homogenized by ultrasonication for $60 \mathrm{~min}$ and centrifuged at $5000 \mathrm{rpm}$ for 30 min at $4^{\circ} \mathrm{C}$. The supernatant was mixed with $1 \mathrm{ml}$ of Ni-NTA agarose solution (Transgene, China) and combined into an affinity column (Sangon, Shanghai, China) at $4^{\circ} \mathrm{C}$ for $10 \mathrm{~min}$. The affinity column was then washed with washing buffer I $\left(20 \mathrm{mM} \mathrm{Na}_{2} \mathrm{HPO}_{4}, 20 \mathrm{mM} \mathrm{NaH} \mathrm{PO}_{4}, 500 \mathrm{mM}\right.$

$\mathrm{NaCl}$, and $5 \mathrm{mM}$ imidazole, $\mathrm{pH}$ 7.8) at 30-fold volume of Ni-NTA agarose solution, and then with washing buffer II (20 mM Na $2 \mathrm{HPO}_{4}, 20 \mathrm{mM} \mathrm{NaH}_{2} \mathrm{PO}_{4}$, and $20 \mathrm{mM}$ imidazole, $\mathrm{pH}$ 7.8) at 15-fold volume of Ni-NTA agarose solution. Washing buffer III (20 mM Na $2 \mathrm{HPO}_{4}, 20 \mathrm{mM} \mathrm{NaH}_{2} \mathrm{PO}_{4}, 500$ $\mathrm{mM} \mathrm{NaCl}$ and $100 \mathrm{mM}$ imidazole, $\mathrm{pH}$ 7.8), washing buffer $\mathrm{IV}\left(20 \mathrm{mM} \mathrm{Na} \mathrm{HPO}_{4}, 20 \mathrm{mM}\right.$ $\mathrm{NaH}_{2} \mathrm{PO}_{4}, 500 \mathrm{mM} \mathrm{NaCl}$ and $150 \mathrm{mM}$ imidazole, $\left.\mathrm{pH} 7.8\right)$ and Washing buffer $\mathrm{V}(20 \mathrm{mM}$ $\mathrm{Na}_{2} \mathrm{HPO}_{4}, 20 \mathrm{mM} \mathrm{NaH}{ }_{2} \mathrm{PO}_{4}, 500 \mathrm{mM} \mathrm{NaCl}$ and $250 \mathrm{mM}$ imidazole, $\mathrm{pH}$ 7.8) were used at 3-fold volume of Ni-NTA agarose solution to wash the Ni-NTA agarose solution in turn, and collected 
219 separately with EP tubes. The imidazole in the eluent was removed using cell washing buffer, and

220 the QseB protein eluent was preserved in $20 \%$ glycerol and stored at $-80^{\circ} \mathrm{C}$ until use. The purity

221 of the QseB protein was analysed by SDS-PAGE and the protein concentration was measured

222 using the Bradford assay with bovine serum albumin (BSA) as a standard.

223

224

225

226

227

228

229

230

231

232

233

234

235

236

237

238

239

\section{Electrophoretic mobility shift assay (EMSA)}

The DNA fragments used for EMSA were obtained by PCR using p-primers from the chromosome of the WT. The p-primers were synthesized by the manufacturer (TsingKE). The p-primers used in EMSA are listed in Table 2. Binding reactions were performed with a total of 150 fmol each probe mixed with various amounts of purified QseB in $4 \mu \mathrm{L} 5 \times$ binding buffer (Beyotime, Shanghai, China) at room temperature for $30 \mathrm{~min}$. Afterward, $5 \mu \mathrm{L}$ gel loading buffer $(0.25 \times \mathrm{TBE}$, $60 \%$; glycerol, 40\%; and bromophenol, $0.2 \%$ (wt/vol)) was added, and mixtures were electrophoresed in a $6 \%$ native polyacrylamide gel in $0.5 \times$ TBE buffer $(45 \mathrm{mM}$ Tris-borate, $1 \mathrm{mM}$ EDTA, $\mathrm{pH}$ 8.0). The result of promoter fragments was purified with a DNA Gel Extraction Kit (Sangon, Shanghai, China), and a chemiluminescent EMSA kit (Beyotime, Shanghai, China) was used to detect the signals of DNA-protein complexes according to the manufacturer's instructions.

\section{Statistical analysis}

All data were analysed using the statistical software SPSS (ver. 19.0, IBM Corp., Armonk, NY) by a one-way ANOVA method; the test results were shown as mean $\pm \mathrm{SD}$. The paired $t$-test was used for statistical comparisons between groups. The level of statistical significance was set at $\mathrm{p} \leq$ 0.05 . 


\section{RESULTS}

\section{Deletion of qseBC did not affect the growth of XL4}

242 The qseBC mutant strain XL4 was generated by $\lambda$ red-mediated recombination. Complementation

243 of the $q s e B C$ mutant was accomplished by expressing the ORF of the $q s e B C$ operon in the pSTV28

244 vector, and strains WT and XL4 were transformed with the empty vector pSTV28. The colony

245 morphologies of XL4/pSTV28 and XL4/pCqseBC on LB agar plates with $16 \mu \mathrm{g} / \mathrm{ml}$

246 chloramphenicol were similar to those of WT/pSTV28. Moreover, the growth curves of

XL4/pSTV28 and XL4/pCqseBC in LB broth with $16 \mu \mathrm{g} / \mathrm{ml}$ chloramphenicol were similar to that of WT/pSTV28 (Fig. 1)

\section{Deletion of the qseBC increased antimicrobial susceptibility}

Antimicrobial susceptibility assays were performed according to the guidelines of the Clinical

Laboratory Standards Institute (CLSI), and the results showed that the WT strain was resistant to

several antibiotics. The MIC values showed that the antibiotic susceptibility of strain XL4/pSTV28

increased in the presence of ciprofloxacin compared with that of strain WT/pSTV28 (Table 3).

These results indicated that QseBC might be associated with the susceptibility of E. coli ECDCM2

to ciprofloxacin. None of the other tested antibiotics showed a change in the MIC when comparing

WT/XL4 strains, suggesting that QseBC is not involved in general AMR but rather has a very

specific effect on a rather limited spectrum of antimicrobials. To further confirm whether or not

deletion of the qseBC affects the survival rates of E. coli strain ECDCM2, antimicrobial activity

assays were performed in E. coli ECDCM2 isogenic derivatives WT/pSTV28, XL4/pSTV28 and

$\mathrm{XL} 4 / \mathrm{pCqseBC}$ in the presence of several antibiotics. The results showed that the survival rates of 
strain XL4/pSTV28 decreased compared with those in the parental strain WT/pSTV28, and the

complemented strain XL4/pCqseBC restored the WT phenotype in the presence of gentamicin and ciprofloxacin (Fig. 2).

Deletion of the qseBC down-regulated the transcription of the multidrug efflux pump activator

marA and efflux pump-associated genes acr $A$, acrB, acrD, emrD and $\mathrm{mdtH}$

It was reported that antibiotic susceptibility to gentamicin and ciprofloxacin are related to efflux

pump-associated genes, namely marA (Perez et al. 2012; Randall \& Woodward 2002) (encoding

the activator of efflux pump transcription), acrA (Nikaido \& Pages 2012) (encoding

the periplasmic lipoprotein component of multidrug efflux pump in E. coli ), acrB (Nikaido \&

Pages 2012) (encoding the substrate: proton antiporter in the inner membrane), acrD (Elkins \&

Nikaido 2002; Rosenberg et al. 2000) (encoding efflux pump of aminoglycosides), emrD (Baker

et al. 2012; Naroditskaya et al. 1993) (encoding a multidrug efflux protein involved in adaptation

to low-energy shock) and $m d t H$ (Nishino \& Yamaguchi 2001) (encoding a multidrug efflux

protein). In this study, we investigated the mechanism of regulation of antibiotic susceptibility to gentamicin and ciprofloxacin by qseBC in E. coli ECDCM2. Firstly, cells of strains WT/pSTV28,

XL4/pSTV28 and XL4/pCqseBC were collected in the middle exponential phase $\left(\mathrm{OD}_{600}=1\right)$, and then the transcript levels of several efflux pump-associated genes, including $\operatorname{mar} A, \operatorname{acr} A, \operatorname{acr} B$, $a c r D$, emrD and $m d t H$ in WT/pSTV28, XL4/pSTV28 and XL4/pCqseBC, were measured by performing real-time reverse transcription PCR experiments. The results showed that the transcript 
282 parental strain WT/pSTV28, and the transcript levels of these genes were restored in

$283 \mathrm{XL4/pCqseBC}$ (Fig. 3). These results suggested that QseBC decreased antibiotic susceptibility by

284 upregulating the transcription of efflux-pump-associated genes $\operatorname{mar} A, \operatorname{acr} A, \operatorname{acr} B, \operatorname{acr} D, \operatorname{emr} D$ and $285 m d t H$.

\section{Deletion of the qseBC decreased biofilm formation}

It has been suggested that biofilm formation is an important factor to evade the poison of antibiotics

and cause persistent infections of the host (Creti et al. 2004; Janssens et al. 2008). In order to

examine the influence of $q s e B C$ on biofilm formation capacity of the $E$. coli strain isolated from a

dairy cow with mastitis, biofilm assays were performed. As shown in Fig. 4A, stained biofilm

adhered to the glass tubes, and the biomass formed by strain XL4/pSTV28 was less than that of

strain WT/pSTV28. The results of quantitative analysis exhibited that the solid-surface-associated

biofilm formation of XL4/pSTV28 decreased 2-fold compared with that of strain WT/pSTV28,

and biofilm formation was partially restored in XL4/pCqseBC (Fig. 4B).

\section{Deletion of the qseBC downregulated the transcription of biofilm-associated genes}

Furthermore, in order to determine the mechanisms of how QseBC affects biofilm formation in

the clinical E. coli strain ECDCM2, the transcript levels of several biofilm-associated genes, including $b \operatorname{cs} A$ (encoding the cellulose synthase catalytic subunit), $\operatorname{csg} A$ (encoding a major subunit of curli fibres), fliC (encoding a basic subunit of the flagellar filament), motA (encoding a component of the flagellar motor complex), wcaF (encoding a putative acetyltransferase involved 
303 levels of $b \operatorname{cs} A, \operatorname{csg} A, f l i C, \operatorname{mot} A, w c a F$ and fimA were downregulated 2.5-fold, 2.8-fold, 1.5-fold,

304 2.0-fold, 1.8-fold and 2.2-fold, respectively, in strain XL4/pSTV28 compared with those in the

305 parental strain WT/pSTV28, and the transcript levels of these genes were restored in strain

306 XL4/pCqseBC (Fig. 5). These data suggested that inactivation of QseBC inhibits biofilm

307 formation in E. coli strain ECDCM2 by downregulating the transcription of biofilm-associated

308 genes $b \operatorname{cs} A, \operatorname{csg} A, f l i C, \operatorname{mot} A, w c a F$ and $\operatorname{fim} A$.

The binding ability assays of QseB to the promoter regions of the target genes

310 Whether QseB binds to the promoters of these genes and directly controls their transcription

311 remains unknown; therefore, we purified a His-tagged QseB to perform electrophoretic mobility

312 shift assay (EMSA). DNA probes containing the putative promoters of several target genes were

313 amplified. A previous study demonstrated that QseB binds directly to its own promoter (Clarke \&

314 Sperandio 2005b). In this study, clearly shifted bands of DNA were observed after incubation of

315 QseB with DNA probes containing the qseB promoter (Fig. 6A), suggesting that the QseB protein

316 we purified has binding affinity for a DNA fragment. EMSA were also performed to detect the

317 binding of QseB to DNA fragments of the promoter regions of marA, acrA, acrD, $\operatorname{mdtH}$ and $e m r D$.

318 As shown in Fig. 6B, the shifted bands of the QseB-marA complex were clearly observed; the

319 intensity of the shifted band was enhanced as the amount of QseB increased, whereas the shifted

320 band disappeared in the presence of an approximately 10 -fold excess of unlabelled promoter DNA

321 fragment as a specific competitor. However, there was no shifted band between QseB and other

322 target genes. These results indicated that QseB can regulate the transcription of marA by binding 323 directly to its promoter region. 


\section{DISCUSSION}

326 Up to now, mastitis has remained a complex disease in the dairy industry worldwide. Moreover, a

previous report indicated that $E$. coli was one of the most frequently isolated pathogens from dairy

cows on large dairy farms (Gao et al. 2017). Multidrug resistance is induced by various interactions

among antimicrobial agents, germs and the environment, and the multidrug efflux pump plays an

indispensable role in driving the wide spread of multidrug resistance. In our study, the $q s e B C$

mutant strain XL4 was more susceptible than the WT strain in the presence of gentamicin and

ciprofloxacin. In addition, the results of RT-qPCR showed that the transcript levels of mar $A$, acr $A$,

$a c r B$, $a c r D$, emrD and $m d t H$ were all downregulated in XL4/pSTV28 compared with those in the

parental strain WT/pSTV28. These results suggested that QseBC decreases antibiotic

susceptibility of the clinical E. coli strain ECDCM2 by upregulating the transcription of efflux-

pump-associated genes $\operatorname{mar} A, \operatorname{acr} A, \operatorname{acr} B, \operatorname{acr} D, \operatorname{emrD}$ and $m d t H$.

Several studies have demonstrated the function of QseB in the regulation of bacterial motility,

adherence and virulence. Clarke and Sperandio demonstrated that QseB binds directly to its own

promoter (Clarke \& Sperandio 2005a). Moreover, QseB could bind the flhDC promoter at high-

340 and low-affinity binding sites directly (Clarke \& Sperandio 2005b). A previous study demonstrated

341 that phosphorylated QseB could bind to the promoter of pilM in A. pleuropneumoniae (Liu et al.

2015). In this study, the EMSA assays were performed to detect the regulatory relationships

between QseB and efflux-pump-associated genes. We found that QseB can bind to the promoter 
344 of marA, which encodes an activator of efflux pump AcrA and AcrB. These results suggested that

345 QseB might regulate the transcription of $a c r A$ and $a c r B$ in a MarA associated pathway.

346 In our study, the biomass of the biofilm formed by the qseBC disruption mutant strain

347 XL4/pSTV28 decreased significantly compared with that formed by strain WT/pSTV28.

348 However, Gou et al. showed that the absence of qseB resulted in increased bacterial motility but

349 no obvious differences in biofilm formation in E. coli MG1655 (Gou et al. 2019). In addition, our

350 results of RT-qPCR showed that inactivation of $q s e B C$ resulted in an apparent decrease in the

351 transcript levels of $\operatorname{css} A, \operatorname{csg} A, f l i C, \operatorname{mot} A, w c a F$ and $\operatorname{fim} A$, whereas Sharma and Casey reported

352 that QseBC did not affect the transcription of $f l i C$, and inactivation of qseBC upregulated the

353 transcription of $\operatorname{csg} A$ in EHEC O157:H7 (Sharma \& Casey 2014). These conflicting results were

354 probably due to the isolation of these E.coli strains from different hosts, and thus the biofilm

355 formation ability and the regulatory mechanisms of biofilm formation might differ between them.

356

357

\section{CONCLUSIONS}

358 The QseBC two-component system affects antibiotic sensitivity by regulating the transcription of the efflux pump activator mar $A$ and the efflux pump-associated genes $a c r A, a c r B, a c r D$, emrD and $m d t H$. Moreover, the EMSA assays demonstrated that QseB can directly bind to the promoter of marA. Furthermore, the biofilm-formation-associated genes $b \operatorname{cs} A, \operatorname{csg} A, f l i C, \operatorname{mot} A, w c a F$ and

fimA were also regulated by QseBC TCS in E. coli ECDCM2. 


\section{Funding}

366 This work was supported by the National Natural Science Foundation of China (grants 31672571)

367 and Graduate Innovation of Anhui Agricultural University (grants 2019yjs-62).

368 Competing Interests

369 The authors report no conflicts of interest in this work.

\section{Author Contributions}

371 Wenchang Li performed the experiments, analyzed the data, prepared figures and/or tables,

372 authored or reviewed drafts of the paper. Mei Xue analyzed the data, contributed

373 reagents/materials/analysis tools. Lumin $\mathrm{Yu}$ and Kezong Qi authored or reviewed drafts of the

374 paper, approved the final draft. Xiaolin Chen, Jingtian Ni and Ruining Deng analyzed the data,

375 prepared figures and/or tables. Fei Shang conceived and designed the experiments, authored or

reviewed drafts of the paper. Ting Xue conceived and designed the experiments, authored or

reviewed drafts of the paper, approved the final draft.

\section{Data Availability}

The following information was supplied regarding data availability:

The raw data are provided in Supplemental Dataset Files.

Baker J, Wright SH, and Tama F. 2012. Simulations of substrate transport in the multidrug transporter EmrD. Proteins 80:1620-1632. 10.1002/prot.24056

Baranova N, and Nikaido H. 2002. The baeSR two-component regulatory system activates transcription of the yegMNOB (mdtABCD) transporter gene cluster in Escherichia coli and increases its resistance to novobiocin and deoxycholate. J Bacteriol 184:4168-4176. 10.1128/jb.184.15.4168-4176.2002 
388 Beier D, and Gross R. 2006. Regulation of bacterial virulence by two-component systems. Curr Opin

389

390

391

392

393

394

395

396

397

398

399

400

401

402

403

404

405

406

407

408

409

410

411

412

413

414

415

416

417

418

419

420

421

422

423

424

425

426

427

Microbiol 9:143-152. 10.1016/j.mib.2006.01.005

Chen X, Shang F, Meng Y, Li L, Cui Y, Zhang M, Qi K, and Xue T. 2015. Ethanol extract of Sanguisorba officinalis L. inhibits biofilm formation of methicillin-resistant Staphylococcus aureus in an icadependent manner. J Dairy Sci 98:8486-8491. 10.3168/jds.2015-9899

Clarke MB, Hughes DT, Zhu C, Boedeker EC, and Sperandio V. 2006. The QseC sensor kinase: a bacterial adrenergic receptor. Proc Natl Acad Sci U S A 103:10420-10425. 10.1073/pnas.0604343103

Clarke MB, and Sperandio V. 2005a. Transcriptional autoregulation by quorum sensing Escherichia coli regulators B and C (QseBC) in enterohaemorrhagic E. coli (EHEC). Mol Microbiol 58:441-455. 10.1111/j.1365-2958.2005.04819.x

Clarke MB, and Sperandio V. 2005b. Transcriptional regulation of flhDC by QseBC and sigma (FliA) in enterohaemorrhagic Escherichia coli. Mol Microbiol 57:1734-1749. 10.1111/j.13652958.2005.04792.x

Creti R, Imperi M, Bertuccini L, Fabretti F, Orefici G, Di Rosa R, and Baldassarri L. 2004. Survey for virulence determinants among Enterococcus faecalis isolated from different sources. $J$ Med Microbiol 53:13-20. 10.1099/jmm.0.05353-0

Datsenko KA, and Wanner BL. 2000. One-step inactivation of chromosomal genes in Escherichia coli K12 using PCR products. Proc Natl Acad Sci U S A 97:6640-6645. 10.1073/pnas.120163297

Dias RS, Eller MR, Duarte VS, Pereira AL, Silva CC, Mantovani HC, Oliveira LL, Silva Ede A, and De Paula SO. 2013. Use of phages against antibiotic-resistant Staphylococcus aureus isolated from bovine mastitis. J Anim Sci 91:3930-3939. 10.2527/jas.2012-5884

Elhadidy M, and Elsayyad A. 2013. Uncommitted role of enterococcal surface protein, Esp, and origin of isolates on biofilm production by Enterococcus faecalis isolated from bovine mastitis. $J$ Microbiol Immunol Infect 46:80-84. 10.1016/j.jmii.2012.02.002

Elkins CA, and Nikaido H. 2002. Substrate specificity of the RND-type multidrug efflux pumps AcrB and AcrD of Escherichia coli is determined predominantly by two large periplasmic loops. $J$ Bacteriol 184:6490-6498. 10.1128/jb.184.23.6490-6499.2002

Gao J, Barkema HW, Zhang L, Liu G, Deng Z, Cai L, Shan R, Zhang S, Zou J, Kastelic JP, and Han B. 2017. Incidence of clinical mastitis and distribution of pathogens on large Chinese dairy farms. $J$ Dairy Sci 100:4797-4806. 10.3168/jds.2016-12334

Gou Y, Liu W, Wang JJ, Tan L, Hong B, Guo L, Liu H, Pan Y, and Zhao Y. 2019. CRISPR-Cas9 knockout of qseB induced asynchrony between motility and biofilm formation in Escherichia coli. Can J Microbiol 65:691-702. 10.1139/cjm-2019-0100

Hoch JA, and Varughese KI. 2001. Keeping signals straight in phosphorelay signal transduction. J Bacteriol 183:4941-4949. 10.1128/jb.183.17.4941-4949.2001

Hughes DT, Clarke MB, Yamamoto K, Rasko DA, and Sperandio V. 2009. The QseC adrenergic signaling cascade in Enterohemorrhagic E. coli (EHEC). PLoS Pathog 5:e1000553. 10.1371/journal.ppat.1000553

Janssens JC, Steenackers H, Robijns S, Gellens E, Levin J, Zhao H, Hermans K, De Coster D, Verhoeven TL, Marchal K, Vanderleyden J, De Vos DE, and De Keersmaecker SC. 2008. Brominated

Peer) reviewing PDF | (2019:10:42311:2:0:NEW 22 Feb 2020) 
428

429

430

431

432

433

434

435

436

437

438

439

440

441

442

443

444

445

446

447

448

449

450

451

452

453

454

455

456

457

458

459

460

461

462

463

464

465

466

467

468 furanones inhibit biofilm formation by Salmonella enterica serovar Typhimurium. Appl Environ Microbiol 74:6639-6648. 10.1128/aem.01262-08

Juarez-Rodriguez MD, Torres-Escobar A, and Demuth DR. 2013. ygiW and qseBC are co-expressed in Aggregatibacter actinomycetemcomitans and regulate biofilm growth. Microbiology 159:9891001. 10.1099/mic.0.066183-0

Khajanchi BK, Kozlova EV, Sha J, Popov VL, and Chopra AK. 2012. The two-component QseBC signalling system regulates in vitro and in vivo virulence of Aeromonas hydrophila. Microbiology 158:259-271. 10.1099/mic.0.051805-0

Liu J, Hu L, Xu Z, Tan C, Yuan F, Fu S, Cheng H, Chen H, and Bei W. 2015. Actinobacillus pleuropneumoniae two-component system QseB/QseC regulates the transcription of PilM, an important determinant of bacterial adherence and virulence. Vet Microbiol 177:184-192. 10.1016/j.vetmic.2015.02.033

Nair MK, Joy J, Vasudevan P, Hinckley L, Hoagland TA, and Venkitanarayanan KS. 2005. Antibacterial effect of caprylic acid and monocaprylin on major bacterial mastitis pathogens. J Dairy Sci 88:3488-3495. 10.3168/jds.S0022-0302(05)73033-2

Naroditskaya V, Schlosser MJ, Fang NY, and Lewis K. 1993. An E. coli gene emrD is involved in adaptation to low energy shock. Biochem Biophys Res Commun 196:803-809. 10.1006/bbrc. 1993.2320

Neave FK, Dodd FH, Kingwill RG, and Westgarth DR. 1969. Control of mastitis in the dairy herd by hygiene and management. J Dairy Sci 52:696-707. 10.3168/jds.S0022-0302(69)86632-4

Nikaido H, and Pages JM. 2012. Broad-specificity efflux pumps and their role in multidrug resistance of Gram-negative bacteria. FEMS Microbiol Rev 36:340-363. 10.1111/j.1574-6976.2011.00290.x

Nishino K, and Yamaguchi A. 2001. Analysis of a Complete Library of Putative Drug Transporter Genes in Escherichia coli. J Journal of Bacteriology 183:5803-5812. 10.1128/JB.183.20.5803-5812.2001

Perez A, Poza M, Aranda J, Latasa C, Medrano FJ, Tomas M, Romero A, Lasa I, and Bou G. 2012. Effect of transcriptional activators SoxS, RobA, and RamA on expression of multidrug efflux pump AcrAB-TolC in Enterobacter cloacae. Antimicrob Agents Chemother 56:6256-6266. 10.1128/aac.01085-12

Randall LP, and Woodward MJ. 2002. The multiple antibiotic resistance (mar) locus and its significance. Res Vet Sci 72:87-93. 10.1053/rvsc.2001.0537

Rosenberg EY, Ma D, and Nikaido H. 2000. AcrD of Escherichia coli is an aminoglycoside efflux pump. J Bacteriol 182:1754-1756. 10.1128/jb.182.6.1754-1756.2000

Saini V, McClure JT, Leger D, Keefe GP, Scholl DT, Morck DW, and Barkema HW. 2012. Antimicrobial resistance profiles of common mastitis pathogens on Canadian dairy farms. J Dairy Sci 95:43194332. $10.3168 /$ jds.2012-5373

Sharma VK, and Casey TA. 2014. Escherichia coli O157:H7 lacking the qseBC-encoded quorum-sensing system outcompetes the parental strain in colonization of cattle intestines. Appl Environ Microbiol 80:1882-1892. 10.1128/aem.03198-13

Weigel WA, and Demuth DR. 2016. QseBC, a two-component bacterial adrenergic receptor and global regulator of virulence in Enterobacteriaceae and Pasteurellaceae. Mol Oral Microbiol 31:379-397. 10.1111/omi.12138

Peer] reviewing PDF | (2019:10:42311:2:0:NEW 22 Feb 2020) 
469 Yang F, Liu LH, Li XP, Luo JY, Zhang Z, Yan ZT, Zhang SD, and Li HS. 2016. Short communication: N$470 \quad$ Acetylcysteine-mediated modulation of antibiotic susceptibility of bovine mastitis pathogens. $J$ $471 \quad$ Dairy Sci 99:4300-4302. 10.3168/jds.2015-10756

472

473

474

475

476 


\section{Table 1 (on next page)}

Strains and plasmids used in this study 
Table 1

\begin{tabular}{|c|c|c|}
\hline $\begin{array}{l}\text { Strains or } \\
\text { plasmids }\end{array}$ & Relevant genotype & $\begin{array}{l}\text { Reference or } \\
\text { source }\end{array}$ \\
\hline \multicolumn{3}{|l|}{ Strains } \\
\hline \multicolumn{3}{|l|}{ E. coli } \\
\hline WT & $\begin{array}{l}\text { E. coli isolated from a dairy cow with mastitis } \\
\text { (ECDCM2), wild-type }\end{array}$ & $\begin{array}{l}\text { Laboratory } \\
\text { stock }\end{array}$ \\
\hline XL4 & ECDCM2 qseBC-deletion mutant & This study \\
\hline $\mathrm{WT} / \mathrm{pSTV} 28$ & WT with the empty vector $\mathrm{pSTV} 28, \mathrm{Cm}^{\mathrm{r} a}$ & This study \\
\hline XL4/pSTV28 & XL4 with the empty vector pSTV28, $\mathrm{Cm}^{\mathrm{r}}$ & This study \\
\hline \multicolumn{3}{|l|}{ Plasmids } \\
\hline pKD46 & $\begin{array}{c}\text { Expresses } \lambda \text { Red recombinase Exo, Bet and Gam, } \\
\text { temperature sensitive, Amp }{ }^{r}\end{array}$ & $\begin{array}{c}\text { (Datsenko \& } \\
\text { Wanner 2000) }\end{array}$ \\
\hline pKD3 & cat gene, template plasmid, $\mathrm{Amp}^{\mathrm{r}} \mathrm{Cm}^{\mathrm{r}}$ & $\begin{array}{l}\text { (Datsenko \& } \\
\text { Wanner 2000) }\end{array}$ \\
\hline pCP20 & $\begin{array}{l}\mathrm{FLP}^{+} \lambda \mathrm{cI} 857^{+} \lambda \mathrm{p}_{\mathrm{R}} \mathrm{Rep}(\mathrm{Ts}), \text { temperature sensitive, } \\
\mathrm{Amp}^{\mathrm{r}} \mathrm{Cm}^{\mathrm{r}}\end{array}$ & $\begin{array}{l}\text { (Datsenko \& } \\
\text { Wanner 2000) }\end{array}$ \\
\hline pSTV28 & Low copy number cloning vector, $\mathrm{Cm}^{\mathrm{r}}$ & Takara \\
\hline pCqseBC & pSTV28 with qseBC gene, $\mathrm{Cm}^{\mathrm{r}}$ & This study \\
\hline
\end{tabular}


Table 2 (on next page)

Oligonucleotide primers used in this study 
1 Table 2

2

\begin{tabular}{|c|c|}
\hline Primer name & Oligonucleotide sequence (5'-3') \\
\hline qseBC-f & GGATTTAACCTTACCAGGCA \\
\hline qseBC-r & GAACCATCATCGCATGTGTG \\
\hline knockout-qseBC-f & $\begin{array}{l}\text { AGAAGATGACATGCTGATTGGCGACGGCATCAAAACG } \\
\text { GGCTGTAGGCTGGAGCTGCTT }\end{array}$ \\
\hline knockout-qseBC-r & $\begin{array}{l}\text { AGACGAGTAGCGCGATCGATCCCGGAATGTAATTGGA } \\
\text { GCATGAATATCCTCCTTAGTTC }\end{array}$ \\
\hline check-qseBC-f & GCGTTACAACACGGTTTACT \\
\hline check-qseBC-r & CGGTACGGTGAAATTAGCAA \\
\hline $\mathrm{Cm}-\mathrm{f}$ & TGTAGGCTGGAGCTGCTT \\
\hline Cm-r & CATATGAATATCCTCCTTAGTTC \\
\hline qseBC-EcoRI-f & CGGAATTCATGCGAATTTTACTGATAGA \\
\hline qseBC-KpnI-r & GGGGTACCTTACCAGCTTACCTTCGTCT \\
\hline M13-f & TGTAAAACGACGGCCAGT \\
\hline M13-r & CAGGAAACAGCTATGACC \\
\hline T7-f & TAATACGACTCACTATAGGG \\
\hline T7-r & TGCTAGTTATTGCTCAGCGG \\
\hline qseB-EcoR I -f & CCGGAATTCATGCGAATTTTACTGATAG \\
\hline qseB-Hind III-r & CCCAAGCTTTCATTTCTCACCTAATGT \\
\hline $\mathrm{rt}-16 \mathrm{~S}-\mathrm{f}$ & TTTGAGTTCCCGGCC \\
\hline rt-16S-r & CGGCCGCAAGGTTAA \\
\hline rt-bcsA-f & GATGGTACAAATCTTCCGTC \\
\hline $\mathrm{rt}-\mathrm{bcsA}-\mathrm{r}$ & ATCTTGGAGTTGGTCAGGCT \\
\hline rt-csgA-f & AGCGCTCTGGCAGGTGTTGT \\
\hline rt-csgA-r & GCCACGTTGGGTCAGATCGA \\
\hline rt-fliC-f & CCTGAACAACACCACTACCA \\
\hline rt-fliC-r & TGCTGGATAATCTGCGCTTT \\
\hline rt-motA-f & GGCAATAATGGCAAAGCGAT \\
\hline rt-motA-r & CAGCGAAAACATCCCCATCT \\
\hline rt-wcaF-f & TCTCGGTGCCGAAAGGGTTC \\
\hline rt-wcaF-r & ATTGACGTCATCGCCGACCC \\
\hline rt-fimA-f & TGCTGTCGGTTTTAACATTC \\
\hline rt-fimA-r & ACCAACGTTTGTTGCGCTA \\
\hline rt-acrA-f & GCAGCCAATATCGCGCAA \\
\hline rt-acrA-r & ATGCGACCGCTAATCGGA \\
\hline rt-acrB-f & TTGCCAAAGGCGATCACG \\
\hline rt-acrB-r & TTGGCAGACGCACGAACA \\
\hline
\end{tabular}




\begin{tabular}{ll}
\hline rt-acrD-f & TGTTCCTGCGTTTGCCGA \\
rt-acrD-r & CATTCGCGCCACGTTTTG \\
rt-marA-f & TGTCCAGACGCAATACTG \\
rt-marA-r & TACGGCTGCGGATGTATT \\
rt-emrD-f & GTATTACTCGTGGCCGTC \\
rt- emrD-r & ATTCCGACGAGGATCACC \\
rt-mdtH-f & GCGAGGAACCTGGGTAAA \\
rt-mdtH-r & CCGCCGAAAATACCCAGA \\
Biotin-p-qseB-f & GTTTATTACTCCCTTTAATG \\
p-qseB-r & TTTTTCATCCCTGCGATAAC \\
Biotin-p-marA-f & TGGTGGTTGTTATCCTGTG \\
p-marA-r & ATTAGTTGCCCTGGCAAGT \\
Biotin-p-acrA-f & ATGTTCGTGAATTTACAGG \\
p-acrA-r & ATGTAAACCTCGAGTGTC \\
Biotin-p-acrD-f & TGCCTCCTACTGACCAAAGAA \\
p-acrD-r & TAAAAGAGGACCTCGTGTTTC \\
Biotin-p-emrD-f & CCGCTTTTGTTTACATAT \\
p-emrD-r & TATCACGGATGCTTTTAT \\
Biotin-p-mdtH-f & TTCCCCTCCCGGGAAATAAA \\
p-mdtH-r & TCTATACCTACTCCTTCCCG \\
\hline
\end{tabular}

3 aThe sequences with the underline refer to the restriction endonuclease recognition sites.

$4 \mathrm{f}=$ forward; $\mathrm{r}=$ reverse.

5

6 
Table 3 (on next page)

Antibiotic susceptibility testing results 
1 Table 3

\begin{tabular}{cccccccccc}
\hline \multirow{2}{*}{ Strains } & \multicolumn{10}{c}{ MIC $(\mu \mathrm{g} / \mathrm{mL})$} \\
\cline { 2 - 10 } & GM & CIP & PN & OF & OXA & KAN & EM & TET & SXT \\
\hline WT/pSTV28 & 2.5 & 4 & 256 & 64 & $>4096$ & 10 & 320 & 100 & $64 / 1216$ \\
XL4/pSTV28 & 2.5 & 2 & 256 & 64 & $>4096$ & 10 & 320 & 100 & $64 / 1216$ \\
XL4/pCqseBC & 2.5 & 4 & 256 & 64 & $>4096$ & 10 & 320 & 100 & $64 / 1216$ \\
\hline
\end{tabular}

2 GM: Gentamicin; CIP: Ciprofloxacin; PN: Penicillin G; OF: Ofloxacin; OXA: Oxacillin; KAN:

3 kanamycin sulfate; EM: Neomycin; TET: Tetracycline; SXT: Paediatric Compound

4 Sulfamethoxazole Tablets

5

6

7 
Figure 1

Growth curves of the wild-type strain WT/pSTV28, the mutant strain XL4/pSTV28, and the complement strain XL4/pCqseBC.

Strains WT/pSTV28, XL4/pSTV28, and XL4/pCqseBC were grown in LB broth with $16 \mu \mathrm{g} / \mathrm{ml}$ chloramphenicol at $37^{\circ} \mathrm{C}$ for $26 \mathrm{~h}$ with shaking, and their growth curves were determined by measuring the cell density (OD) at $600 \mathrm{~nm}$.

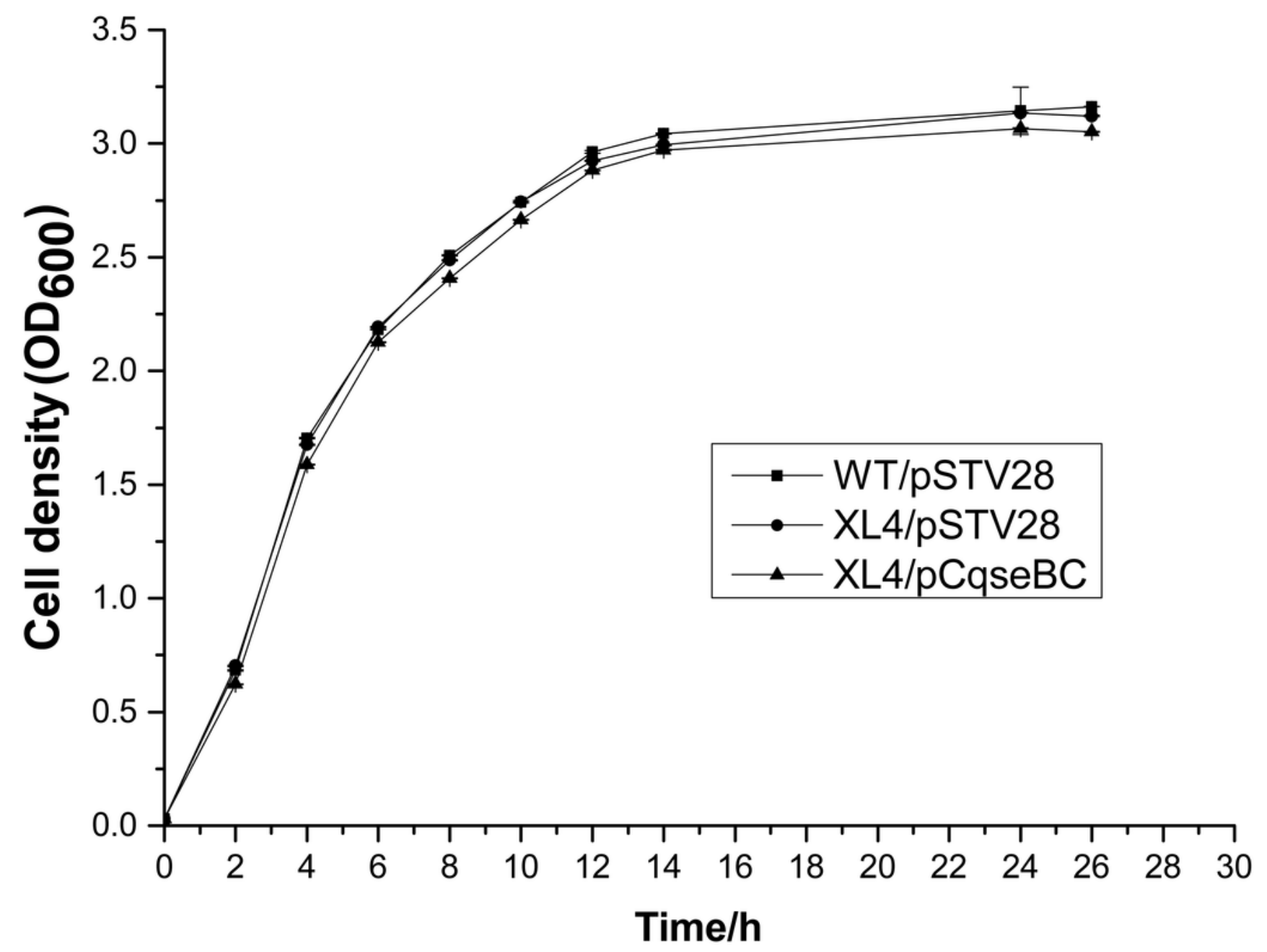




\section{Figure 2}

Antimicrobial activity assay of $E$. coli strain isogenic derivatives WT/pSTV28, XL4/pSTV28 and XL4/pCqseBC cultured with different antibiotic conditions.

The survival rate of the parental strain was designated as $100 \%$. The colony counts of XL4/pSTV28 with different antibiotics (A: ciprofloxacin; B: gentamicin) were compared with that of the parental strain WT/pSTV28. Error bars indicate standard deviations. $* * P<0.01$, means difference in WT/pSTV28, XL4/pSTV28 and XL4/pCqseBC.
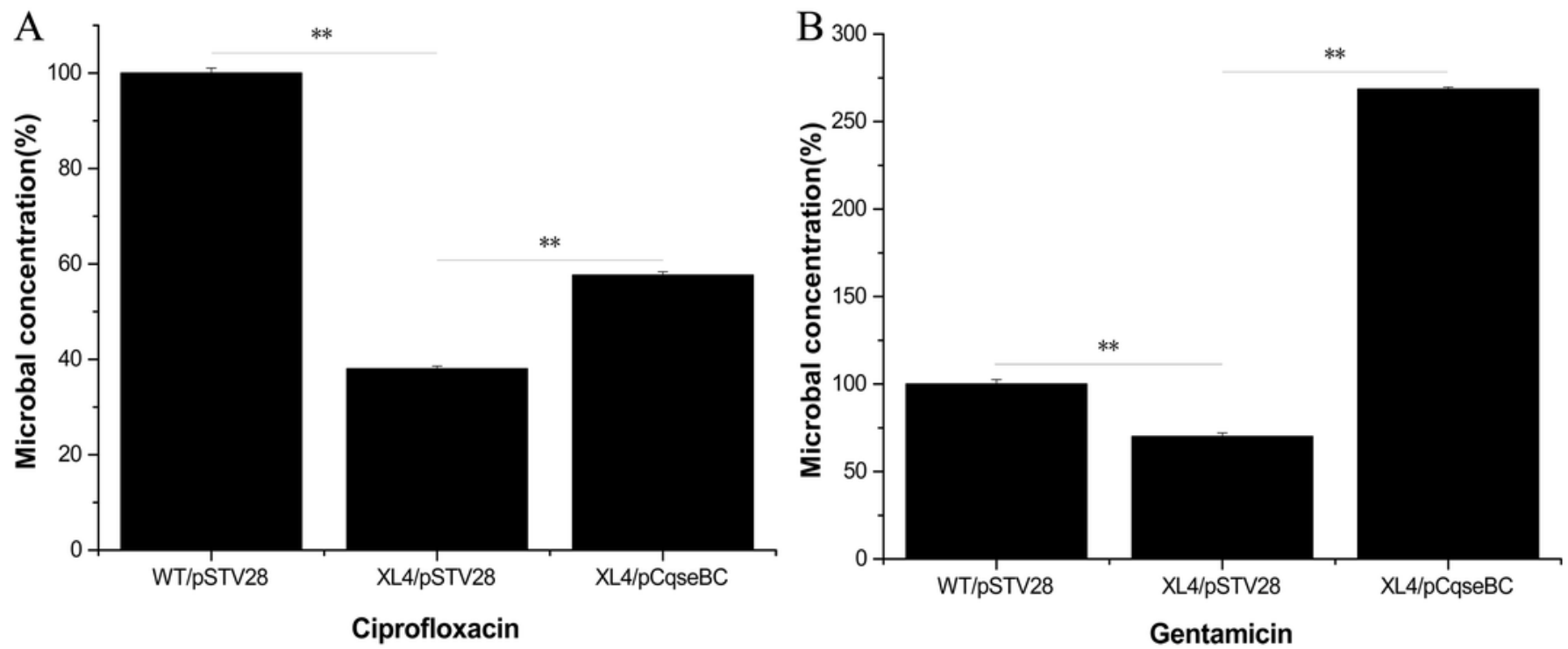


\section{Figure 3}

Fluctuation of transcript levels of efflux-pump-associated genes.

Real-time reverse transcription PCR experiments were utilized to detect the transcript levels of efflux-pump -associated genes marA, acrA, acrB, acrD, emrD and mdtH in strains WT/ pSTV28, XL4/pSTV28 and XL4/pCqseBC incubate in LB broth with $16 \mu \mathrm{g} / \mathrm{ml}$ chloramphenicol. Double asterisks $(* * P<0.01)$ and single asterisk $(* P<0.05)$ means difference in WT/pSTV28, XL4/pSTV28 and XL4/pCqseBC.

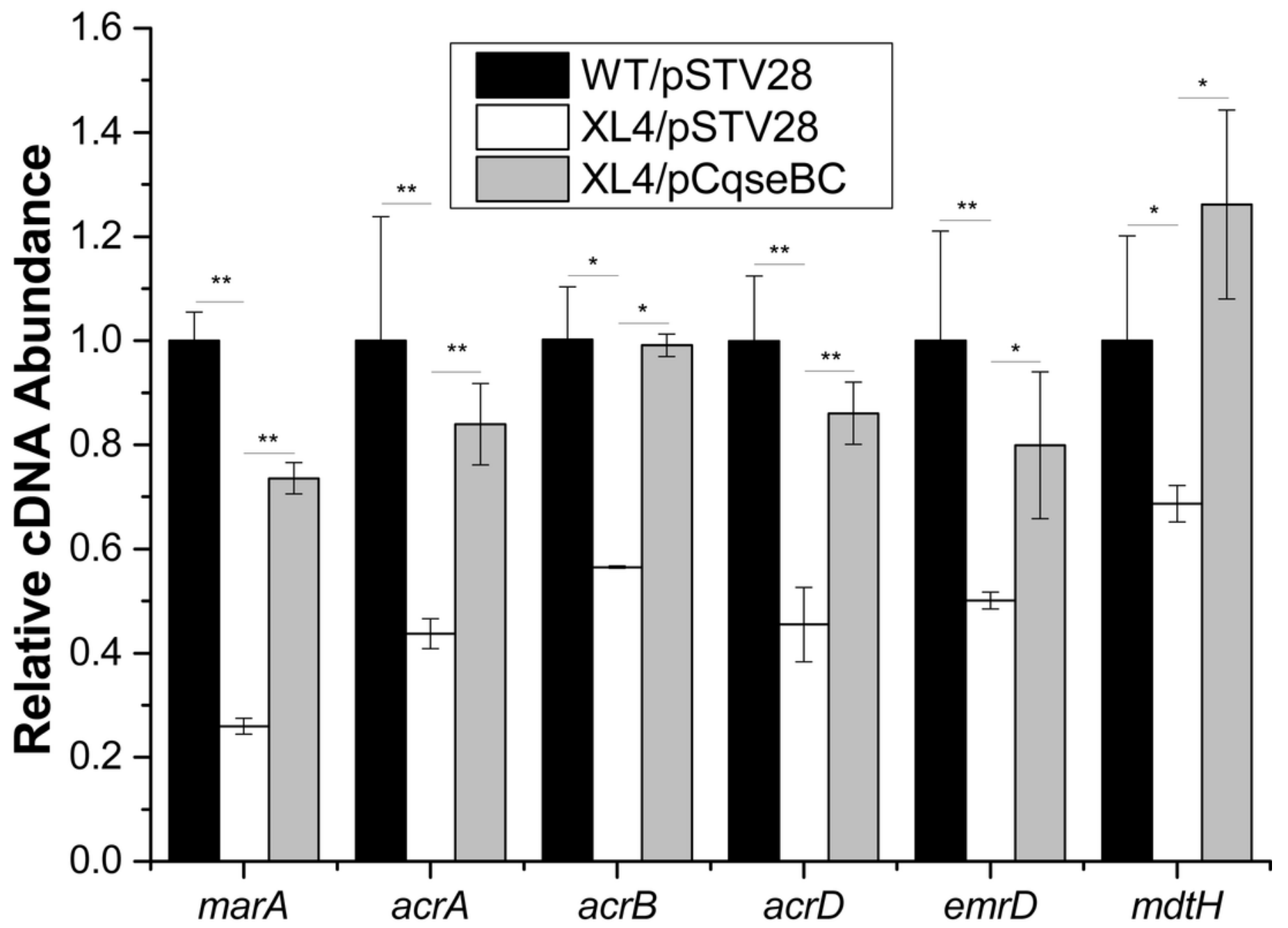




\section{Figure 4}

Detection of biomass of biofilm formed by WT/pSTV28, XL4/pSTV28 and XL4/pCqseBC on glass tubes by $\mathrm{CV}$ staining.

Biofilms were quantified after $72 \mathrm{~h}$ incubation at $37{ }^{\circ} \mathrm{C}$ without shaking. (A) Image of biofilm stained with $1 \% \mathrm{CV}$ and adhered on glass tubes. (B) Biofilm were stained by $1 \% \mathrm{CV}$ and dissolved the purple area with $33 \%$ glacial acetic acid and measured by optical density at a wavelength of $492 \mathrm{~nm}$. Error bars indicate standard deviations. Double asterisks ( $* * P<$ 0.01) means difference in WT/pSTV28, XL4/pSTV28 and XL4/pCqseBC.

A

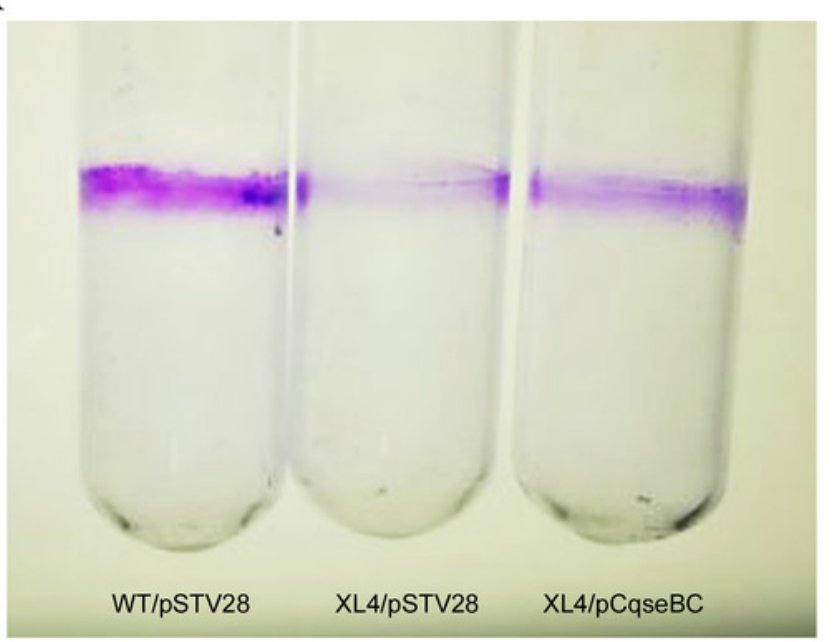

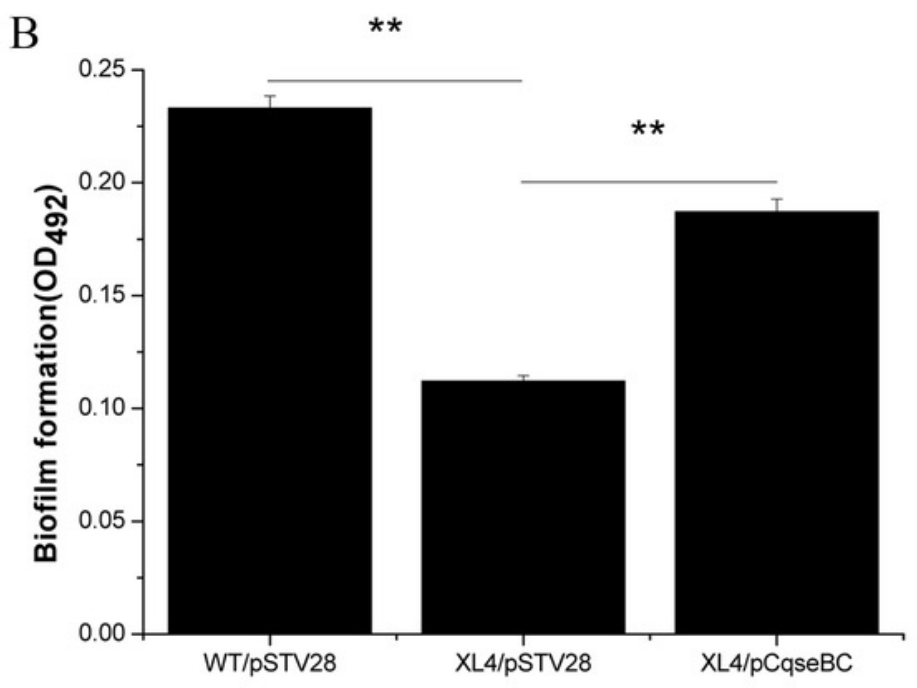




\section{Figure 5}

Result of transcript levels of biofilm-associated genes.

Real-time reverse transcription PCR experiments were utilized to detect the transcript levels of biofilm-associated genes bcsA, csgA, flic, motA, wcaF, and fimA in strains WT/ pSTV28, XL4/pSTV28 and XL4/pCqseBC incubate in LB broth with $16 \mu \mathrm{g} / \mathrm{ml}$ chloramphenicol. Double asterisks $(* * P<0.01)$ and single asterisk $(* P<0.05)$ means difference in WT/pSTV28, XL4/pSTV28 and XL4/pCqseBC.

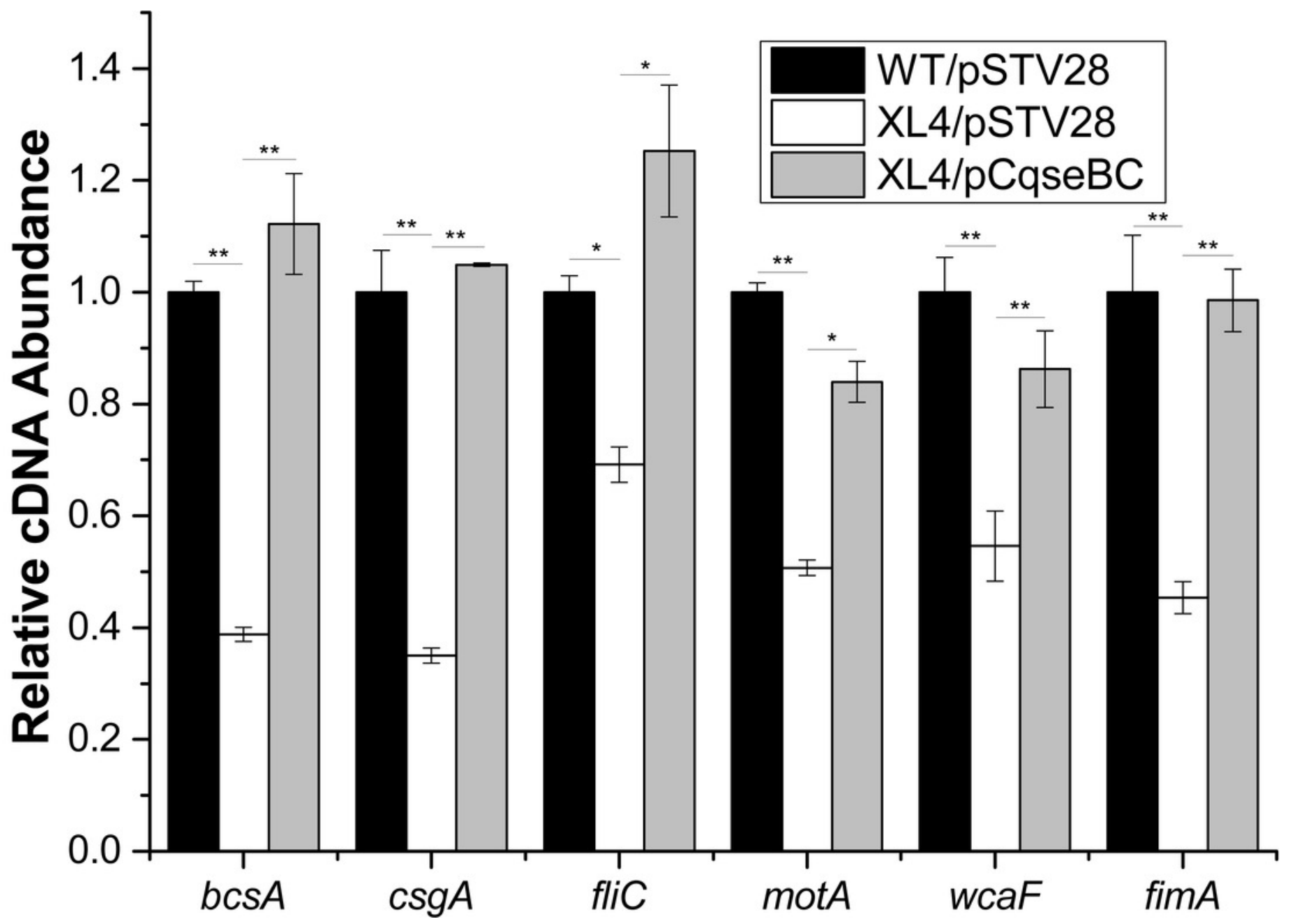




\section{Figure 6}

Electrophoretic mobility shift assay for QseB.

Increasing amounts of QseB were incubated with Biotin-labeled qseB, marA, acrA, acrD, $m d t H$ and emrD promoters (Biotin-p-qseB, Biotin-p-marA, Biotin-p-acrA, Biotin-p-acrD, Biotinp- $m d t H$, Biotin-p-emrD). The lanes 1 to 5 were the DNA probe with increasing amounts of QseB (12, 0,3,6 and $12 \mu \mathrm{M}) ; 150 \mathrm{fmol}$ of Biotin-labeled probes was added to all lanes. And additional $1.5 \mathrm{pmol}$ of unlabeled probe was added in lane 1 as the competitive control (Ctrl). A. The positive control, demonstrating the binding ability of QseB to the qseB promoter; B. the marA promoter; $\mathrm{C}$. the acrA promoter; D. the acrD promoter; $\mathrm{E}$. the $\mathrm{mdtH}$ promoter; $\mathrm{F}$. the emrD promoter. 

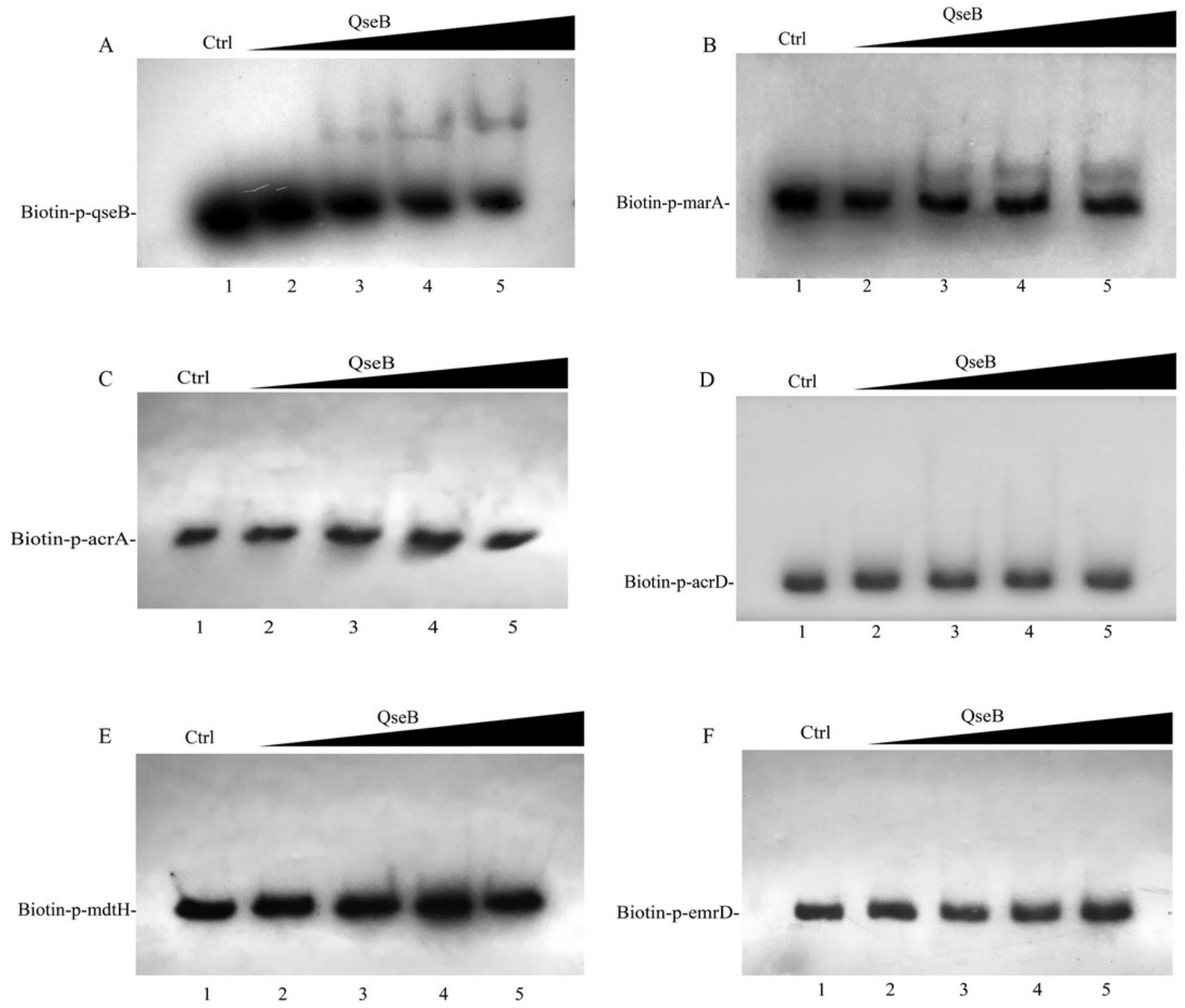